\title{
A propos de la balance à chocolat ou: où commence la médecine?
}

\section{Eberhard Wolff}

PD Dr rer. soc., rédacteur culture, histoire, société

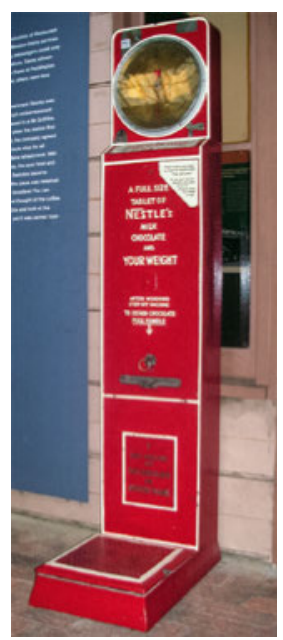

Ma piscine couverte préférée comporte deux pèse-personnes publics qui permettent de connaître son poids lorsque l'on y introduit une pièce. Un modèle plus ancien, galbé, analogique, avec un mécanisme à aiguille. Et un autre plus récent, rectangulaire, avec l'affichage LED du siècle dernier, qui semble déjà archaïque. Je n'ai encore jamais vu personne se peser dessus. Aujourd'hui, on se pèse à la maison.

De telles balances se sont multipliées dans les gares, lors des foires ou dans les pharmacies durant l'ère industrielle. Des tableaux affichés dessus devaient aider à déterminer le «bon" poids, autrement dit le poids «sain». La médecine étendait ses longs bras dans la vie courante, dans les gares et les foires.

C'était l'époque où les gens et leur corps commençaient à être soumis plus souvent à des mesures et des tests. Le thermomètre médical avait fait son apparition. Bientôt, se peser en public était devenu si courant que le «Times» en parlait par exemple comme d'une «vieille coutume anglaise» en 1956 [1]. En 2004 déjà, Enrico Danieli évoquait dans cette revue les balances publiques des gares suisses, qui crachaient le résultat sur un petit billet de carton [2].

Dans les années 1930, le fabricant britannique de balances Salter a mis sur le marché un pèse-personne public (voir photo) qui, au lieu d'accompagner le résultat d'une exhortation à mener une vie saine, offrait une tablette complète de chocolat au lait Nestlé produit en Grande-Bretagne! Ce qui ressemble aujourd'hui à un poisson d'avril était à l'époque tout sauf absurde. On ne se pesait pas, comme si souvent aujourd'hui, dans l'angoisse des kilos superflus. Certes, cette crainte se répandait déjà à l'époque où les balances publiques se sont popularisées. Mais la balance à chocolat n'a pu avoir du succès que parce qu'en ce temps-là, les gens se pesaient souvent simplement pour s'amuser. Ou par curiosité, envie d'en apprendre davantage sur leur corps. Et quelquesuns aussi, parce qu'ils auraient bien aimé peser plus.

Et même s'ils visaient leur poids idéal, ce n'était (et ce n'est) bien souvent pas seulement pour être en bonne santé, mais aussi par souci esthétique. Durant la période qui a marqué l'essor des balances publiques, l'idéal de beauté a également évolué dramatiquement vers la minceur.

La pesée s'étend(ait) à de nombreux domaines, dont quelques-uns seulement liés à la santé.

Bébé, j'ai été, comme beaucoup de Suissesses et Suisses d'un certain âge, pesé sur une balance très spéciale: elle était dotée d'un accessoire plat, recourbé vers le haut sur les côtés, qui servait à peser la progéniture. Un autre accessoire, en forme de plat creux celui-là, la transformait en balance de cuisine pour peser la farine du gâteau du dimanche.

Une balance de cuisine avec fonction bébé ou une balance pour bébé avec fonction cuisine? Les deux. Dans le même esprit, notre balance à chocolat n'est ni un distributeur de chocolat pesant les gens ni un pèsepersonne distribuant du chocolat, mais les deux à la fois.

Et la balance à chocolat comme la balance de cuisine pèse-bébé sont pour moi de beaux exemples du fait que la médecine n'est pas un domaine fermé. Elle ne commence pas brutalement lorsqu'on entre dans un cabinet médical ou un hôpital, pas plus qu'elle ne s'arrête lorsqu'on en sort. Il y a un peu de médecine dans chaque foire où les gens peuvent se peser. Et il y a toujours un peu de "foire» en médecine. Ou pour le formuler un peu plus sérieusement: la médecine et sa logique convergent en de nombreux points avec d'autres domaines de la vie courante qui suivent une autre logique. Au premier abord, la balance à chocolat nous semble bizarre. En réalité, elle est la norme.

\section{Crédit photo}

Grace's Guide to British Industrial History

\section{Références}

1 Bivins R, Marland H. Weighting for Health. Management, Measurement and Self-surveillance in the Modern Household Social History of Medicine 2016;29:757-80.

2 Danieli E. Vom Messen und Wiegen. Bulletin des médecins suisses 2004;85:2272-3. 